\title{
Particle Physics with Low Cost SiPM Based Detectors
}

\author{
Brett Fadem ${ }^{1}$ \\ ${ }^{1}$ Physics Department, Muhlenberg College, 2400 Chew Street, Allentown, PA, USA
}

\begin{abstract}
Muon counting experiments provide students with an opportunity to learn about particle detection in high energy physics, detector design/construction, electronics, coincidence counting, counting statistics, and characteristics of the muons that traverse our bodies every minute of every day. The goal of this project is to construct a muon detector sensitive to the direction of muons, make a measurement of rate vs. angle, and compare the result both to the known angular distribution and to the results of a computer simulation. Several aspects of the design are left open: The first amplification stage of the signal and the digitization stage can be designed by the students. These activities use mailing tubes as the basis for the detectors, but students might choose to design their own mechanical structures and enclosures. If they so choose, students have the opportunity to work with CAD software, 3D printing, and laser cutting. Another selling point for the design described here is the use of silicon photomultipliers (SiPMs) instead of traditional high voltage photomultiplier tubes, providing students with exposure to modern light detection techniques in high energy physics. Finally, while not described in these proceedings, the associated activity of creating a computer simulation of muon rates vs. angle helps students to understand the physical considerations that affect the measurement they are making.
\end{abstract}

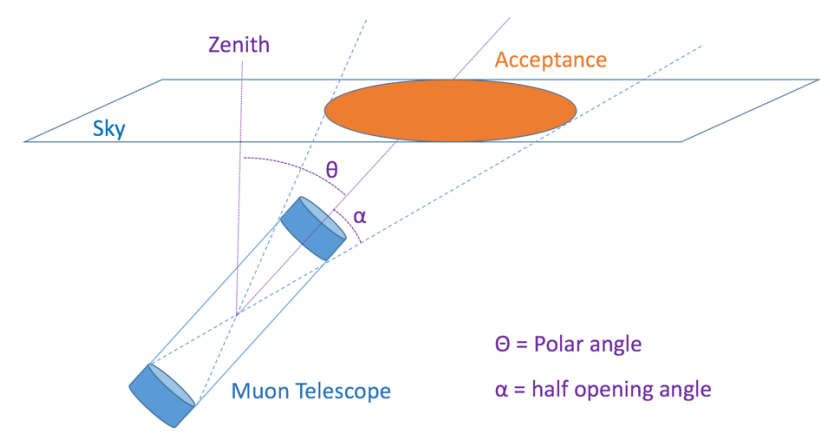

FIG. 1. The detecting medium of the muon telescope consists of two plastic scintillating cylinders placed in line as shown. The angle from zenith, $\theta$, and geometric acceptance in terms of the half opening angle, $\alpha$ are also shown in the figure.

\section{INTRODUCTION}

Many nuclear and particle physics experiments require the use of radioactive samples, but a subset of these use muons, electrically charged particles whose properties are similar to electrons but whose mass is about 200 times greater (or about $106 \mathrm{MeV} / \mathrm{c}^{2}$ ) [1]. Muons are among the particles created in collisions between cosmic rays and atoms in Earth's atmosphere. Although they typically decay after about 2.2 microseconds in their own reference frame, their relativistic speeds cause them to persist for much longer times in the reference frame of the earth, and the presence of muons created in the upper atmosphere at sea level is evidence of time dilation. When all is said and done, the rate of muons at sea level from all directions is about $1 \mathrm{~cm}^{-2}$ minute $^{-1}$ [2]. The experiment we describe involves measuring muon rates as a function of angle from the zenith, $\theta$, see Fig. 1 . There is a wellknown cosine squared angular dependence to this rate [2]. The experimental goal of these activities is to measure this dependence using a muon telescope.

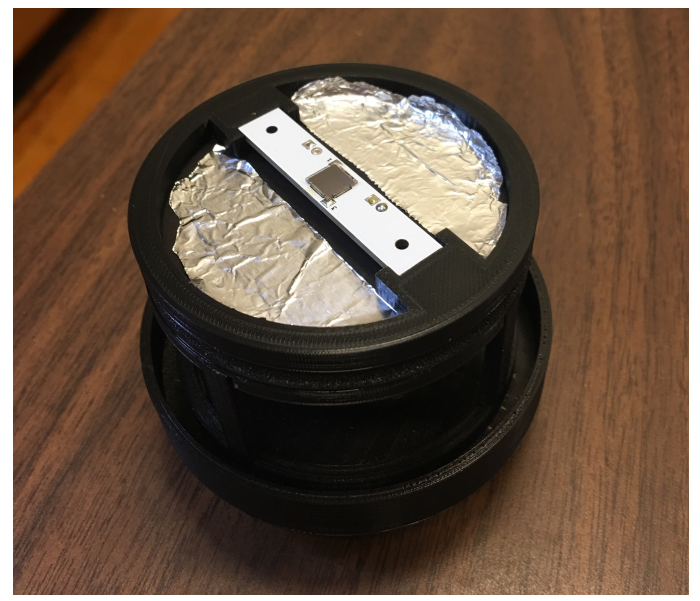

FIG. 2. A silicon photomultiplier (SiPM), SiPM printed circuit board (SiPM PCB) and SiPM PCB holder are shown, as well as a foam core disk coated with aluminum foil, and the rest of the mechanical assembly.

To create our muon telescope, we place two muon counters in an enclosure. The heart of each muon counter is a scintillating plastic cylinder mated to a silicon photomultiplier (SiPM). Our enclosure consists of a dirt cheap length of a mailing tube with a three inch inner diameter. Figure 1 shows a cartoon of the two plastic scintillators in the tube. In Fig. 2, a SensL SiPM requiring a reverse bias voltage of only 29.4 volts is soldered to a SiPM printed circuit board (SiPM PCB) designed by S.N. Axani, J.M. Conrad, and C. Kirby [3]. Also visible in the figure is a 3D printed SiPM PCB holder, and a laser cut foam core disk covered by aluminum foil. These form part of the Muhlenberg counter assembly. The aluminum foil on the foam core disk reflects back some of the light that escapes through the bottom of the scintillator, and the foam core disk itself helps to optically isolate the scintillator. The counter assembly with scintillator attached is shown in Fig. 3. By considering coincidences (nearly si- 
multaneous hits in the two scintillators) we limit ourselves to muons whose trajectory intersects both scintillators and constrain the geometric acceptance of the detector. One muon counter is placed inside each end of the mailing tube. The longer the mailing tube, the further apart the two scintillating cylinders, and the smaller the angular acceptance of the device.

Two muon telescopes were demonstrated at the workshop. One has a tube length of $61.4 \mathrm{~cm}$, a half opening angle of $\alpha=9.7$ degrees and is shown in Fig 4. The data shown in Fig. 8 are from this telescope. As shown in the figure, the rate when vertical is $15.1 \mathrm{mHz}$ and thus the average time between coincidences is just over a minute. The other muon telescope demonstrated at the workshop had a shorter tube in which the two scintillator assemblies were placed as close together as possible (while remaining optically isolated). The coincidence rate on the shorter tube muon telescope was about 0.3 $\mathrm{Hz}$ when vertical, so workshop participants only had to wait a few seconds between coincidences.

In addition to the SiPM PCB described above, the detectors we have built use an additional printed circuit board (PCB) design created by S. N. Axani, J. M. Conrad, and C. Kirby for further processing of the signal [3]. Their paper provides detailed schematics for both PCBs and instructions on how to build a detector they have designed. The result is a compact and elegant product. The goals for our activities are slightly different. Rather than striving for this elegant end-product, we wish to open things up so that students can more easily make changes to the design. Accordingly, we implement some of the electronics on a solderless breadboard and some of our electronic design differs from theirs. Another significant point of departure in the design is that, in ours, a single detector consists of two scintillating counters rather than one so that we can easily create our aligned muon telescope.

\section{DETECTOR DESIGN}

Our detectors are based on plastic scintillator, a material that is inexpensive and easy to handle. The type we use, BC412 , is a general purpose scintillator often used for time-offlight measurements of charged particles such as muons. The 3 inch diameter by 1.5 inch long cylindrical shapes we use are currently readily available on ebay for about $\$ 50 /$ scintillator. Interestingly, the mailing tube design for the detector was inspired by the fact that the scintillator once arrived in a mailing tube. When charged particles traverse the plastic scintillator, light is produced. The scintillator is then placed against a light sensor, such as a traditional photomultiplier tube, or, in our case, a SiPM, which is a solid state stand-in for the traditional photomultiplier. The counter assembly is shown in Fig. 3.

The SiPM is soldered to the SiPM PCB, and optical grease is applied at the interface between the scintillator and the SiPM. Soldering surface mount components is a significant aspect of the project, and the author has employed both sol-

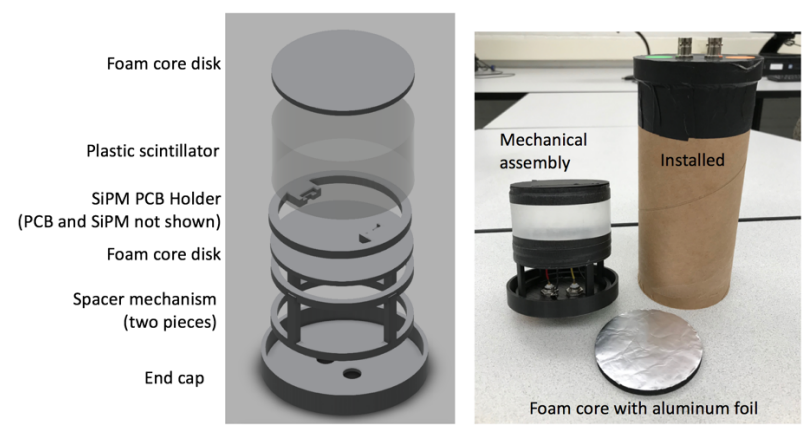

FIG. 3. An exploded view of the design of the mechanical assembly is shown at left, and its realization is shown at right, along with the smaller muon telescope containing one installed assembly.

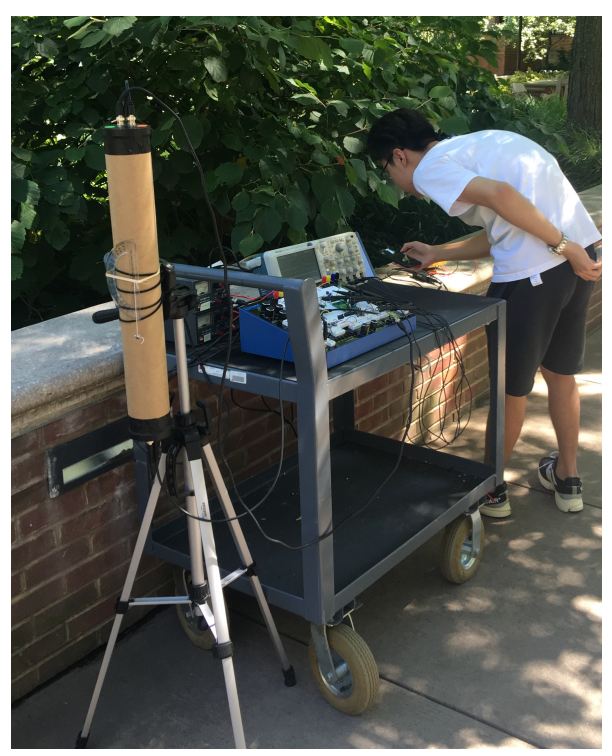

FIG. 4. One of the completed detectors used for the BFYIII conference mounted on a tripod.

dering iron and reflow soldering techniques. The signal and reverse bias voltage lines are routed from the SiPM PCB to BNC bulkhead connectors on the end caps using small wires. On the other side of the end cap, the BNC bulkhead connectors and coaxial cables route the signal to the breadboard and the reverse bias voltage power supply. The design of the counters provides mechanical support for the wiring. The design shown in Fig. 3 was created using Autodesk Fusion 360. Most parts were then 3D printed on site at Muhlenberg College but the foam core disks were produced using a laser cutter from a maker space in Allentown, PA, Make Lehigh Valley. The longer of the two completed telescopes for the BFYIII conference is shown in Fig. 4. 


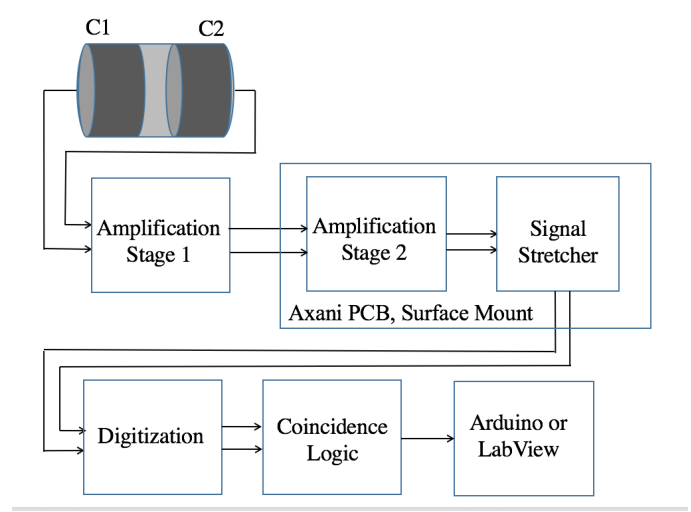

FIG. 5. The overall layout for the detector electronics is shown above. A mailing tube contains two scintillating counters, $\mathrm{C} 1$ and $\mathrm{C} 2$. The signals from these are sent to two amplification stages, one on the breadboard and a second on the Axani electronics PCB. The PCB also contains a circuit to stretch the signals out in time, followed by a digitization stage, coincidence detecting stage that combines signals from both counters, and data acquisition with either an Arduino or a National Instruments DAQ device (controlled by LabView) and a PC.

\section{ELECTRONICS AND SOFTWARE}

The electronics on the breadboard follows the logical flow in Fig. 5. The first stage of the electronics consists of a noninverting op amp amplifier implemented using through hole components. The op amp itself consists of the same chip as is used on the electronics PCB, an LT6201 dual op amp, but it is mounted on a breakout board with headers so that it can be plugged directly into the breadboard. Ordinary carbon resistors can be used for the amplification. This gives the instructor a chance to ask the students to design their own first stage of amplification before using the electronics PCB. The students will be prompted to design the first stage amplifier with a nominal gain of 10 , and they should expect that their amplifiers will approach that performance. However, there is often a significant DC offset imposed by the first stage of amplification, and we have used a blocking capacitor to remove this offset after the first stage. This attenuates the first stage output slightly, but poses no overall problem. The output of the first stage amplification is routed to the Axani electronics PCB, where it is further amplified and then conveyed to a "peak detecting" circuit (as it is referred to in [3]) which converts the short raw pulse (about $0.5 \mu \mathrm{s}$ long) to a longer one (a few hundred $\mu$ s long). Thus, it is essentially a signal stretching circuit. While these parts of the Axani electronics PCB are used, it isn't fully populated with components. The SiPM reverse bias voltage of 29.4 volts is pulled from an external supply, so we don't populate the Axani electronics PCB with one, and while we use an Arduino Nano to process coincidences at the end of the electronics chain, we don't place Nanos on each Axani electronics PCB as prescribed. Instead, we route the output of the "peak detecting" (or "signal

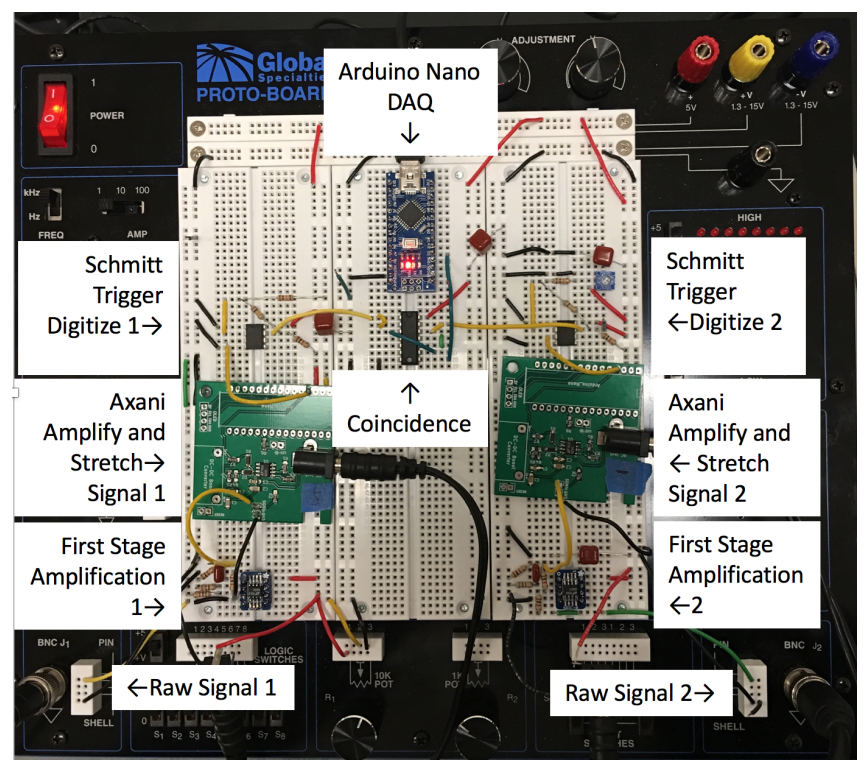

FIG. 6. Realization of the electronics from amplification of the raw signals to the coincidence logic on a quad NAND gate, and data acquisition with an Arduino Nano communicating coincidences to a $\mathrm{PC}$ via a virtual COM port. A python script runs on the PC to record the PC time stamp for each coincidence.

stretching") circuit on the Axani electronics PCB to a Schmitt Trigger on the breadboard, bypassing the spot on the Axani electronics PCB for a Nano. The adjustable trigger level on the Schmitt trigger in the subsequent stage can accommodate any idiosyncrasies of the amplification process.

Just as students will be asked to design the first stage of amplification, they will be prompted to design the Schmitt trigger using a 311 comparator and through hole components. The Schmitt trigger will act both as a discriminator (an important function in nuclear and particle physics) and a digitizer for the signals. The venerable 311 comparator is slow by modern standards but it is inexpensive and ubiquitous. It causes a slight glitch in the original signal in our detector but we use it to demonstrate that once the signal has been stretched (by the "peak detecting" circuit on the Axani electronics PCB) it is easy to manipulate with electronic components common in the undergraduate laboratory. Finally, students will be asked to design the coincidence logic, implemented with a Quad NAND gate, that combines the digitized signals from each counter.

A realization of the electronics described here is shown in Fig. 6. The Arduino Nano is used to communicate signals over a virtual serial port to a PC, where a small Python script time stamps each count with the PC time and writes the count and timestamp information to a text file. The uncertainty in the PC timestamp is small relative to the hours one must typically wait to accumulate data at each angle, so one need not be concerned about its limited precision.

Oscilloscope traces for the raw signal, the stretched and amplified signal, and digitized signal are shown in Fig. 7. It 


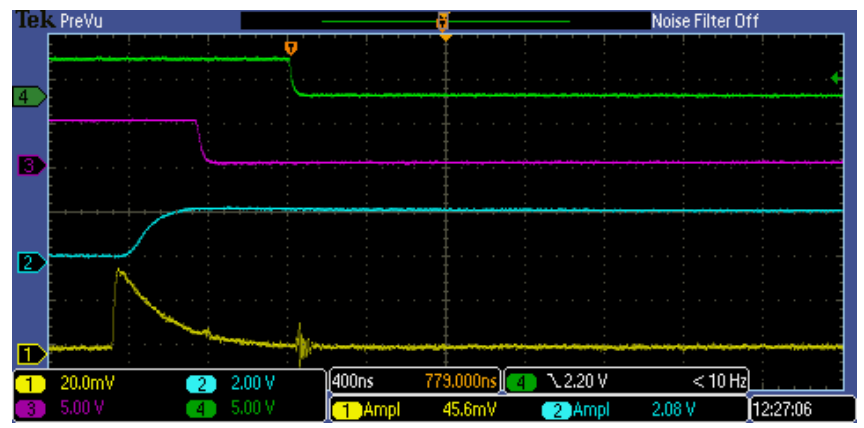

FIG. 7. Oscilloscope traces, running from the bottom of the screen to the top, for the raw signal (yellow), amplified and stretched signal (cyan), and digitized signal (magenta) and coincidence signal (green) are shown.

should be noted that a similar version of some of these electronics and data acquisition techniques were used in combination with GM tube detectors last spring in the author's advanced laboratory class. Students enjoyed developing the electronics to process the signals.

\section{RESULTS}

Results of the experiment using the muon telescope described in these proceedings are shown in Fig. 8. A scaled cosine squared curve is provided for comparison. The error bars in the plot are statistical. For each angle measured, the detector ran until 100 counts were accumulated. According to Poisson statistics, the uncertainty in the counts is equal to the square root of the number of counts, so each measurement at a given polar angle had a 10 percent uncertainty. The total number of counts was divided by the time it took to acquire them, and this is the source of the rates shown in the plot. Since the half opening angle was nearly 10 degrees, the data bins in these results are not all entirely independent.

\section{CONCLUSIONS}

Muon detectors using modern, solid state technology have been built inexpensively (the most expensive items are the SiPM light sensors at \$120/each, and the scintillators, which are currently about $\$ 50 /$ each). Soldering tools have repre- sented an extra cost for us, representing a one-time expense of under $\$ 1000$. Since the conference, we have successfully designed and milled our own PCBs as an alternative to the Axani PCBs described in these proceedings. The author is open to the idea of producing populated PCBs for institutions interested in trying these activities but without interest in the PCB aspects.

The project provides opportunities for students to use the electronics they have learned in a scientific setting, to gain experience with CAD tools, 3D printing and laser cutting, and to

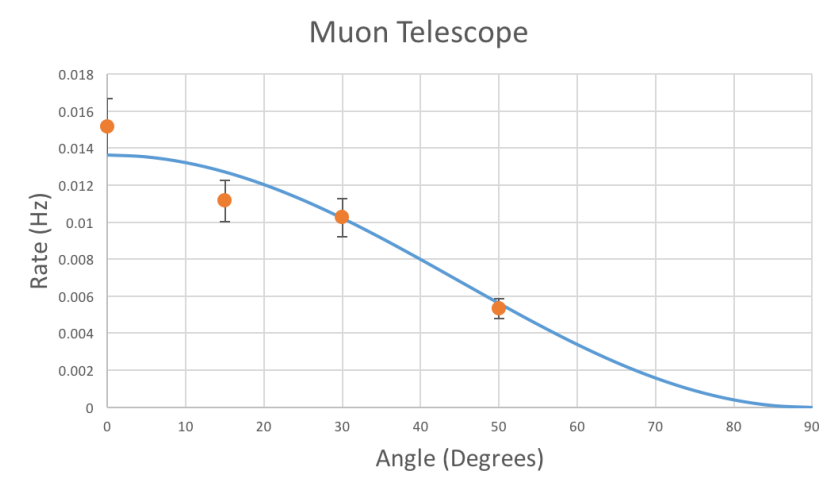

FIG. 8. Results of the rate vs. angle experiment. The error bars reflect a 10 percent statistical uncertainty on each data point, and the half angle, $\alpha$, of the muon telescope acceptance is 9.7 degrees. A scaled cosine squared function is shown for comparison.

obtain an important introduction to experimental techniques in high energy physics. The activities are quite open ended, and the computer simulation exercise provides the students with a tool to understand their experimental results. While there has not been a discussion of this computer simulation exercise in these proceedings, the author intends to publish information on the simulation in the future.

\section{ACKNOWLEDGMENTS}

The author wishes to acknowledge NSF grant 1507841, and the assistance of his many students including Hongwen Yu, Meredith Jones, Lucas Kasle, Richard Morash, Kelly Tornetta, Travis Crawford, and Charles Bene. I also wish to thank Joseph Kozminski for his help and collaboration, and Merritt Jacob for his consistent support.
[1] http://pdg.lbl.gov/2018/listings/rpp2018-list-muon.pdf. Retrieved $7 / 15 / 18$

[2] http://pdg.lbl.gov/2018/reviews/rpp2018-rev-cosmic-rays.pdf, p. 6 , Retrieved 7/15/18

[3] S. N. Axani, J. M. Conrad, and C. Kirby, "The desktop muon de- tector: A simple, physics-motivated machine- and electronicsshop project for university students," Am. J. Phys. 85, 948 (2017) 